\title{
DINÂMICA POPULACIONAL DE MOSCAS-DAS-FRUTAS (Diptera: Tephritidae) EM POMAR DE GOIABA NO MUNICÍPIO DE JABOTICABAL - SP
}

\author{
Rogério Teixeira Duarte ${ }^{1}$; Júlio César Galli²; Wilson Carlos Pazini ${ }^{3}$. \\ ${ }^{1}$ Doutorando em Agronomia, Faculdade de Ciências Agrárias e Veterinárias de Jaboticabal, Universidade Estadual \\ Paulista Júlio de Mesquita Filho (UNESP), Jaboticabal, São Paulo, Brasil, rogerioteixeira_1@ hotmail.com \\ ${ }^{2}$ Professor Doutor da Faculdade de Ciências Agrárias e Veterinárias de Jaboticabal, UNESP, Jaboticabal, São Paulo, \\ Brasil,jcgalli@fcav.unesp.br \\ ${ }^{3}$ Doutor, Engenheiro Agrônomo da UNESP, Jaboticabal, São Paulo, Brasil, wpazini@ @cav.unesp.br
}

\begin{abstract}
RESUMO: A importância sobre o monitoramento e a dinâmica populacional de espécimes de moscas-das-frutas (Diptera: Tephritidae) influencia substancialmente na tomada de decisão e sobre a escolha dos métodos de controle preconizados pelo Manejo Integrado de Pragas. Dessa forma, os objetivos da pesquisa foram monitorar e analisar a dinâmica populacional de adultos de moscasdas-frutas e sua correlação com os elementos meteorológicos em pomar de goiaba no município de Jaboticabal-SP. A pesquisa foi realizada em um pomar de goiaba entre outubro de 2007 e janeiro de 2009. O monitoramento populacional de adultos de moscas-das-frutas foi realizado com a instalação de cinco armadilhas modelo Mc Phail ${ }^{\circledR}$, com atrativo alimentar composto pelo produto comercial Moscatex $^{\circledR}(3 \%)$ e suco açucarado de goiaba (50\%). A densidade populacional de adultos de moscas-das-frutas foi analisada em histogramas e correlacionada com os dados meteorológicos de temperatura máxima $\left({ }^{\circ} \mathrm{C}\right)$, temperatura mínima $\left({ }^{\circ} \mathrm{C}\right)$, temperatura média $\left({ }^{\circ} \mathrm{C}\right)$, umidade relativa média $(\%)$ e precipitação pluviométrica acumulada $(\mathrm{mm})$. Os resultados obtidos permitiram concluir que $C$. capitata apresentou menor predominância, quando comparada ao gênero Anastrepha, com maior densidade populacional entre os meses de outubro e dezembro de 2007, com picos populacionais nos meses de outubro e novembro de 2007. Não houveram correlações significativas entre as populações de moscas-das-frutas e os elementos meteorológicos.
\end{abstract}

PALAVRAS-CHAVE: Anastrepha spp., Ceratitis capitata, manejo integrado de pragas.

\section{POPULATION DYNAMICS OF FRUIT FLIES (Diptera: Tephritidae) IN GUAVA ORCHARD IN JABOTICABAL - SP}

\begin{abstract}
The importance about monitoring and population dynamics of fruit flies species (Diptera: Tephritidae) influence substantially in decision and on the choice of control methods recommended by Integrated Pest Management. In this way, the goals of this research were monitor and analyze the population dynamics of adults of fruit flies and your correlation with the meteorological elements in guava orchard in Jaboticabal - SP. The research was conducted in guava orchard between October 2007 and January 2009. The population monitoring of adults of fruit flies was performed with five Mc Phail ${ }^{\circledR}$ traps, with food bait consisting commercial product Moscatex ${ }^{\circledR}$ $(3 \%)$ and sweet guava juice (50\%). The population density of fruit flies was analyzed in histograms and correlated with the meteorological dates maximum temperature $\left({ }^{\circ} \mathrm{C}\right)$, minimum temperature $\left({ }^{\circ} \mathrm{C}\right)$, mean temperature $\left({ }^{\circ} \mathrm{C}\right)$, relative humidity $(\%)$ and cumulative rain precipitation $(\mathrm{mm})$. The results showed that $C$. capitata had lower prevalence when compared to the genus Anastrepha, with higher population density from October until December 2007 and reaches the top in October and November 2007. There were not significant correlations between the population of fruit flies and meteorological elements.
\end{abstract}

KEY-WORDS: Anastrepha spp., Ceratitis capitata, integrated pest management. 


\section{INTRODUÇÃO}

A produção de frutas frescas no Brasil é considerada uma das maiores do mundo (SOUZA FILHO et al., 2000), com destaque para o cultivo da goiaba (RAGA et al., 2006), principalmente das regiões Nordeste e Sudeste (MOURA; MOURA, 2006; COLOMBI; GALLI, 2009). O mercado brasileiro de frutas enfrenta sérios problemas relacionados à área fitossanitária, com destaque às moscas-das-frutas, que comprometem substancialmente o comércio de frutas com o exterior devido às restrições quarentenárias impostas pelos países importadores (DUYCK et al., 2004).

A família Tephritidae é composta por mais de 5.000 espécies de moscas-das-frutas, sendo muitas vezes consideradas pragas primárias de muitas espécies de frutíferas (NASCIMENTO; CARVALHO, 2000). Por apresentar característica cosmopolita e polífaga, estas espécies estão difundidas em grande parte do globo terrestre, e concentradas nas regiões de clima tropical e temperado. No Brasil, as espécies de moscasdas-frutas consideradas pragas de importância econômica para a fruticultura se enquadram principalmente no gênero Anastrepha (Shiner, 1868) (Diptera: Tephritidae), além da espécie Ceratitis capitata (Wiedeman, 1824) (Diptera: Tephritidae) (NASCIMENTO; CARVALHO, 2000; MARTINS, 2002).

Como forma de garantir a qualidade da produção com consequente redução no nível populacional destes tefritídeos, o controle químico ainda é o método mais aplicado pela maioria dos produtores, sendo que parte dos agrotóxicos utilizados não apresentam registro no Ministério da Agricultura, Pecuária e Abastecimento. Uma das alternativas para diminuir o uso de agrotóxicos sem comprometer a produtividade é a utilização de programas do Manejo Integrado de Pragas (MIP). Para isso, as pragas e os inimigos naturais devem ser monitorados diretamente nas folhas, frutos ou por meio de armadilhas (PARAJULEE; SLOSSER, 2003).

A flutuação populacional de moscasdas-frutas apresenta grande variabilidade em abundância e épocas de ocorrência de picos populacionais ao longo dos anos (RONCHITELES; SILVA, 2005). Dentre os fatores que interferem nesta variação, os principais envolvem a sucessão de hospedeiros primários ou alternativos, a complexidade do ambiente e os fatores abióticos (CANESIN; UCHÔA-FERNANDES, 2007; MONTES et al., 2011).

A região de Jaboticabal caracteriza-se por ser um dos principais municípios produtores de goiaba no estado de São Paulo (COLOMBI; GALLI, 2009). Entretanto, poucos estudos sobre a dinâmica populacional das moscas-das-frutas têm sido realizados neste município, constituindo-se como um entrave na tomada de decisão sobre as técnicas de controle a serem adotadas no contexto da implementação de programas de MIP. 
Em vista disso, esta pesquisa objetivou monitorar e analisar a dinâmica populacional de adultos de moscas-das-frutas e sua correlação com os elementos meteorológicos em um pomar de goiaba no município de Jaboticabal-SP.

\section{MATERIAL E MÉTODOS}

A pesquisa foi realizada em um pomar de goiaba localizado na Fazenda Experimental da Faculdade de Ciências Agrárias e Veterinárias - UNESP - Campus de Jaboticabal $\left(21^{\circ} 15^{\prime} 18^{\prime \prime} \mathrm{S}\right.$ e $48^{\circ} 19^{\prime} 19^{\prime \prime} \mathrm{W}$, e altitude de $605 \mathrm{~m}$ ), entre outubro de 2007 e janeiro de 2009, com amostragens quinzenais ao longo deste período.

A área monitorada foi de aproximadamente 0,5 hectares do cultivar Paluma, com idade média de 16 anos e espaçamento $7,0 \mathrm{~m} \times 5,0 \mathrm{~m}$. O pomar foi mantido sem plantas espontâneas durante todo o período da pesquisa, com poda de limpeza dos ramos velhos e doentes realizada nos meses de julho de 2007 e 2008.

O tratamento fitossanitário constou de uma única aplicação de parathion methyl (Folisuper $60^{\circledR}$ ), em outubro de 2008, na dosagem de $100 \mathrm{~mL}$ produto comercial/100 L de água, para o controle do besouro rendilhador Costalimaita ferruginea (Fabr., 1801) (Coleoptera: Chrysomelidae).

O monitoramento populacional de adultos de moscas-das-frutas foi realizado com armadilhas modelo Mc Phail ${ }^{\circledR}$. O atrativo alimentar utilizado no interior das mesmas foi composto por suco açucarado de goiaba a $50 \%$ adicionado do produto comercial Moscatex $^{\circledR}$ 3\%. O suco de goiaba foi preparado conforme a metodologia proposta por Rampazzo (1994), utilizando-se a polpa de seis frutos de goiaba fervida em água e açúcar na proporção $400 \mathrm{~mL}$ de água e 200 $\mathrm{mg}$ respectivamente. Depois de fervida, esta mistura foi peneirada e acondicionada em garrafas de $500 \mathrm{~mL}$, sendo armazenadas em refrigerador. No momento do preparo das soluções, o conteúdo de cada garrafa (500 $\mathrm{mL}$ ) foi diluído em água formando um litro de suco (50\%), no qual foi adicionado $300 \mathrm{~mL}$ de Moscatex ${ }^{\circledR}(3 \%)$.

No interior do pomar foram instaladas cinco armadilhas, a uma altura de 1,5 metros do solo, em pontos tomados aleatoriamente no talhão e com disposição para o lado sul (S), como forma de atenuar a incidência direta dos raios solares. As armadilhas foram mantidas no campo por 15 dias, sendo o atrativo alimentar renovado após uma semana. As mesmas foram recolhidas e transportadas ao laboratório de Seletividade Ecológica do Departamento de Fitossanidade da FCAV-UNESP para realização da triagem dos insetos pelo processo de hidropeneiração.

Os dados relacionados às amostragens de adultos de moscas-das-frutas foram analisados em histogramas e correlacionados com os dados meteorológicos de temperatura máxima $\left({ }^{\circ} \mathrm{C}\right)$, temperatura mínima $\left({ }^{\circ} \mathrm{C}\right)$, temperatura média $\left({ }^{\circ} \mathrm{C}\right)$, umidade relativa média $(\%)$ e precipitação pluviométrica $(\mathrm{mm})$, 
que foram registrados pelo Posto Meteorológico da FCAV-UNESP - Campus de Jaboticabal-SP, situado a cerca de $2.500 \mathrm{~m}$ da área. Para os valores de temperatura e umidade relativa foram calculadas médias dos

\section{RESULTADOS E DISCUSSÃO}

A maior representatibilidade populacional de adultos de moscas-das-frutas esteve relacionada principalmente ao gênero Anastrepha, com um total de 4.210 indivíduos amostrados, que correspondeu a 86,64\% do total de tefritídeos coletados. A captura de $C$. capitata foi de 649 espécimes, correspondendo a $13,36 \%$ do total de moscasdas-frutas. Esta relação também foi evidenciada por Raga et al. (2006), Canesin e Uchôa-Fernandes (2007), Alberti et al. (2009), em que o gênero Anastrepha compreendeu a maior parcela de moscas-dasfrutas amostradas por armadilhas com utilização de atrativos alimentares. De acordo com Araújo et al. (2005), Da Silva et al. (2007) a goiaba é considerada o hospedeiro primário do gênero Anastrepha, corroborando os resultados apresentados.

A densidade populacional de adultos de moscas-das-frutas de Anastrepha spp. foi representada por duas grandes variações (Figura 1). A primeira ocorreu entre a segunda quinzena de outubro ao início de dezembro de 2007, com pico populacional no início de novembro, com 536 adultos capturados nas cinco armadilhas com atrativo alimentar. Estes resultados estão de acordo
15 dias anteriores à amostragem, e para precipitação foi utilizado o valor acumulado neste período.

com Ronchi-Teles e Silva (2005) e Chavarria et al. (2009), cujas maiores densidades populacionais de adultos de moscas-das-frutas do gênero Anastrepha estiveram compreendidas entre as estações da primavera e do verão.

A segunda variação ocorreu a partir do final de janeiro estendendo-se até o início de abril de 2008, com maior número de indivíduos coletados no mês março, compreendendo dois picos populacionais, o primeiro no começo do mês, com 579 adultos amostrados, e o segundo no final do mês, com 575 indivíduos coletados em cinco armadilhas com atrativo alimentar para cada data amostral (Figura 1). Souza et al. (2008), concluíram que a maior densidade populacional do gênero Anastrepha esteve compreendida entre os meses de abril a setembro, mesmo apresentando disponibilidade de frutos na área durante todo o ano, discordando com os resultados apresentados.

A maior densidade populacional de adultos de $C$. capitata ocorreu entre a segunda quinzena de outubro ao início de dezembro de 2007, correspondendo em dois picos populacionais (Figura 2). O primeiro na 
segunda metade do mês de outubro, com 233 indivíduos capturados, e o segundo no início de novembro, com 235 adultos amostrados. Resultados diferentes foram obtidos por Moura e Moura (2006), que observaram aumento no número de indivíduos capturados da espécie $C$. capitata a partir do mês de dezembro, com pico populacional em janeiro.

A elevada densidade populacional de adultos do gênero Anastrepha e da espécie $C$. capitata entre os meses de outubro e dezembro de 2007 esteve relacionada provavelmente devido à presença de frutos maduros de outras espécies frutíferas, presentes no entorno do pomar de goiaba analisado.

A flutuação populacional de adultos de Anastrepha spp. compreendida entre os meses de janeiro e abril de 2008, esteve relacionada principalmente à presença de frutos em maturação ou maduros na área de goiaba (Figura 1). O aumento populacional de moscas-das-frutas na presença de frutos maduros também foi observado por Chavarria et al. (2009) e Montes et al. (2011).

A baixa densidade populacional de moscas-das-frutas, a partir da segunda metade de 2008 até início de 2009, pode ter estar relacionada a aplicação do inseticida de ingrediente ativo parathion methyl, utilizado no controle do besouro rendilhador $C$. ferruginea. A utilização de produtos químicos, de baixa seletividade, pode resultar no controle de outras pragas e também de inimigos naturais, que acarreta em uma mudança drástica no equilíbrio biológico de uma determinada área ou cultivo. Este fato foi observado por Pazini e Galli (2011) que obtiveram baixa captura de espécimes de tefritídeos em pomar de goiaba que recebeu aplicações de inseticidas.

A correlação entre a densidade populacional de adultos de moscas-das-frutas com os dados meteorológicos não apresentaram resultados significativos. Estes resultados estão de acordo com os relatados por Corsato (2004), em áreas tropicais, onde a flutuação de adultos de moscas-das-frutas está relacionada principalmente à presença de outras espécies frutíferas consideradas hospedeiras de moscas-das-frutas e não às variáveis climáticas. Corroboram também com os resultados de Canesin e UchôaFernandes (2007) que relataram que os fatores climáticos não foram os únicos determinantes da flutuação populacional das espécies mais frequentes de Anastrepha spp, como a disponibilidade de hospedeiros alternativos, inimigos naturais e sucessão hospedeira.

Com relação à precipitação, os resultados obtidos corroboram com os encontrados por Celedonio-Hurtado et al. (1995), que verificaram que a densidade populacional de moscas-das-frutas não se correlacionou com a precipitação pluviométrica. Analogamente, Ronchi-Teles e Silva (2005) relataram que a ocorrência de moscas-das-frutas não está relacionada com a elevação da precipitação pluviométrica, pois mesmo com outras condições favoráveis, o aumento da umidade 
do solo pode influenciar na diminuição de pupários e contribuir para um menor nível populacional durante as amostragens.

A correlação entre a temperatura média e a flutuação populacional de moscas-das-frutas não significativa também foi constatada em pesquisa realizada por Feitosa et al. (2008). Entretanto, Garcia et al. (2003) encontraram correlação significativa entre a temperatura média e umidade relativa do ar com os níveis populacionais de moscas-das-frutas, divergindo dos resultados apresentados, principalmente, pelo fato de que o experimento foi instalado em uma região considerada sub-tropical.

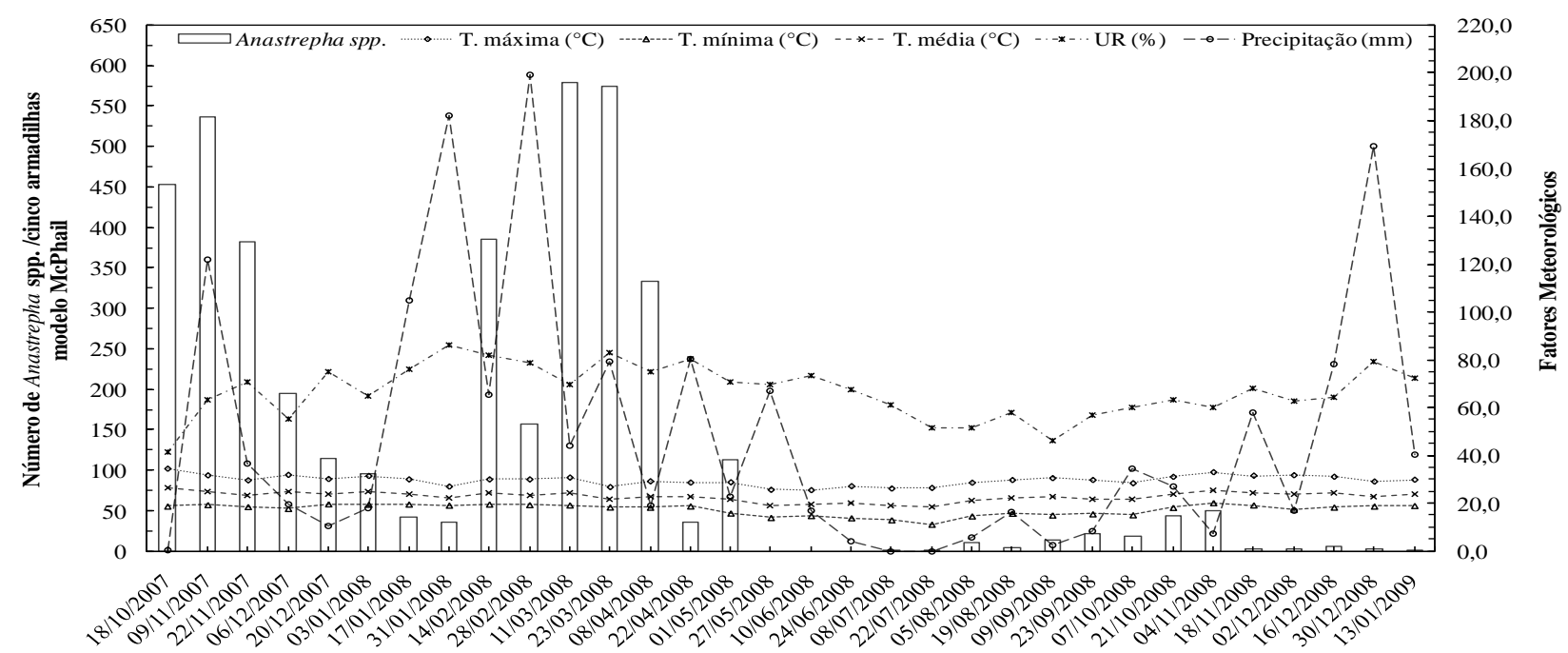

Figura 1. Dinâmica populacional de Anastrepha spp. e fatores metereológicos em pomar de goiaba. Jaboticabal - SP. Temperatura máxima $\left({ }^{\circ} \mathrm{C}\right)$ [T. máxima $\left.\left({ }^{\circ} \mathrm{C}\right)\right]$; Temperatura mínima $\left({ }^{\circ} \mathrm{C}\right)[\mathrm{T}$. mínima $\left.\left({ }^{\circ} \mathrm{C}\right)\right]$; Temperatura média $\left({ }^{\circ} \mathrm{C}\right)\left[\mathrm{T}\right.$. média $\left.\left({ }^{\circ} \mathrm{C}\right)\right]$; Umidade Relativa Média (\%) [U.R. (\%)]; Precipitação Pluviométrica Acumulada (mm) [Precipitação (mm)].

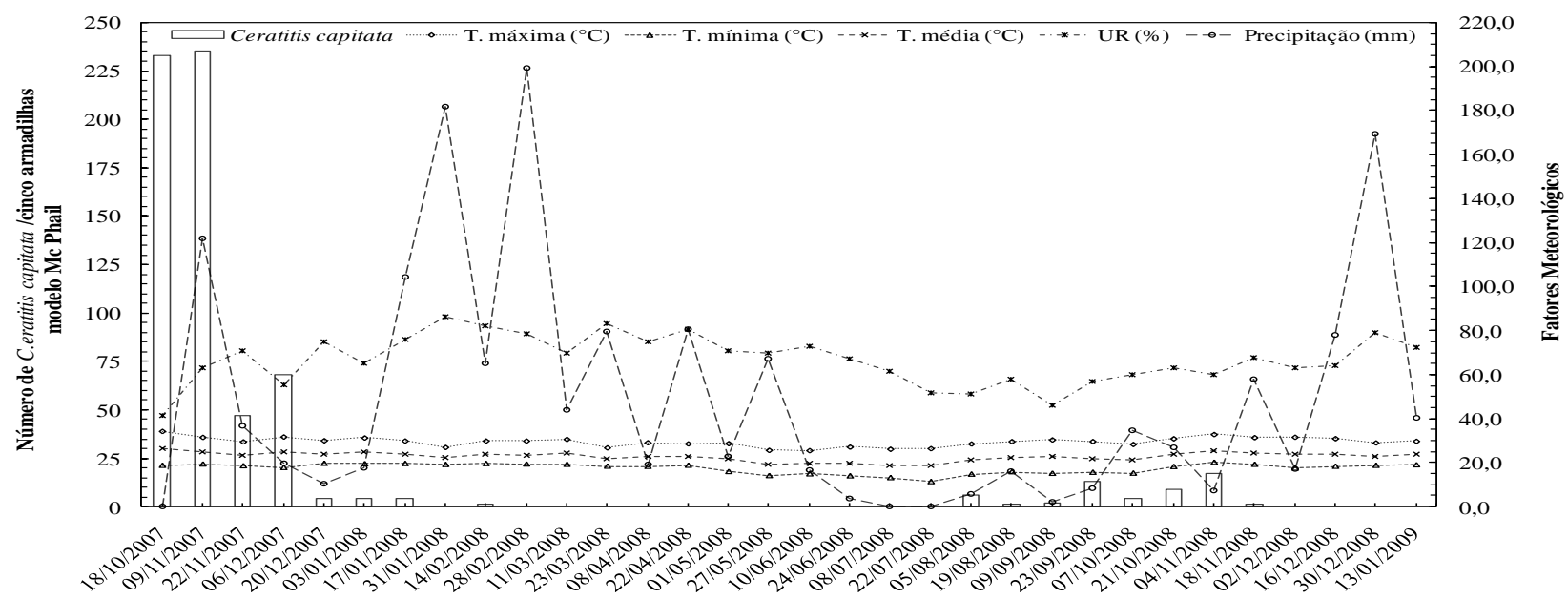

Figura 2. Dinâmica populacional de Ceratitis capitata e fatores metereológicos em pomar de goiaba. Jaboticabal - SP. Temperatura máxima $\left({ }^{\circ} \mathrm{C}\right)\left[\mathrm{T}\right.$. máxima $\left.\left({ }^{\circ} \mathrm{C}\right)\right]$; Temperatura mínima $\left({ }^{\circ} \mathrm{C}\right)$ [T. mínima $\left.\left({ }^{\circ} \mathrm{C}\right)\right]$; Temperatura média $\left({ }^{\circ} \mathrm{C}\right)\left[\mathrm{T}\right.$. média $\left.\left({ }^{\circ} \mathrm{C}\right)\right]$; Umidade Relativa Média $(\%)$ [U.R. (\%)]; Precipitação Pluviométrica Acumulada (mm) [Precipitação (mm)]. 


\section{CONCLUSÃO}

C. capitata apresentou menor predominância, quando comparada ao gênero Anastrepha, com maior densidade populacional entre os meses de outubro e dezembro de 2007, com picos populacionais nos meses de outubro e novembro de 2007.

Não houve correlações significativas entre as populações de moscas-das-frutas e os dados meteorológicos.

\section{REFERÊNCIAS}

ALBERTI, S.; GARCIA, F. R. M.; BOGUS, G. M. Moscas-das-frutas em pomares de pessegueiro e maracujazeiro, no município de Iraceminha, Santa Catarina, Brasil. Ciência Rural, v.39, n.5, p.1565-1568, 2009.

ARAÚJO, E. L.; MEDEIROS, M. K. M.; SILVA, V. E.; ZUCCHI, R. A. Moscas-dasfrutas (Diptera: Tephritidae) no semi-árido do Rio Grande do Norte: plantas hospedeiras e índices de infestação. Neotropical Entomology, v.34, n.6, p.889-894, 2005.

CANESIN, A.; UCHÔA-FERNANDES, M. A. Análise faunística e flutuação populacional de moscas-das-frutas (Diptera, Tephritidae) em um fragmento de floresta semidecídua em Dourados, Mato Grosso do Sul, Brasil. Revista Brasileira de Zoologia, v.24, n.1, p.185-190, 2007.

CELEDONIO-HURTADO, H.; ALUJA, M. R.; LIEDO, P. F. Adult population fluctuations of Anastrepha species (Diptera:

Tephritidae) in tropical orchard habitats of Chiapas, Mexico.

Environmental

Entomology, v.24, n.4, p.861-869, 1995.

CHAVARRIA, G.; ZART, M.; BOTTON, M.; DOS SANTOS, H. P.; MARODIN, G. A. B. Flutuação populacional de adultos de Anastrepha fraterculus (Wied.) em cultivo protegido e convencional de videira. Revista Brasileira de Fruticultura, v.31, n.3, p.725731, 2009.

COLOMBI, C. A.; GALLI, J. C. Dinâmica populacional e evolução de dano de Triozoida limbata (Hemiptera: Psillydae) em goiabeira, em Jaboticabal - SP. Ciência e Agrotecnologia, v.33, n.2, p.412-416, 2009.

CORSATO, C. D. A. Moscas-das-frutas (Diptera: Tephritidae) em pomares de goiaba no norte de Minas Gerais: Biodiversidade, Parasitóides e Controle Biológico. 2004. 95f. Tese (Doutorado) Escola Superior de Agricultura "Luiz de Queiroz", Universidade de São Paulo, Piracicaba, 2004.

DA SILVA, R. A.; DO NASCIMENTO, D. B.; DE DEUS, E. G.; DE SOUZA, G. D.; DE OLIVEIRA, L. P. S. Hospedeiros e parasitóides de Anastrepha spp. (Diptera: Tephritidae) em Itaubal do Piririm, Estado do Amapá, Brasil. Ciência Rural, v.37, n.2, p.557-560, 2007.

DUYCK, P. F.; DAVID, P.; QULICI, S. A review of relationships between interspecific 
competition and invasions in fruit flies (Diptera: Tephritidae). Ecological Entomology, v.29, n.5, p.511-520, 2004.

FEITOSA, S. S.; SILVA, P. R. M.; PÁDUA, L. E. M.; CARVALHO, E. M. S.; PAZ, J. K. S.; PAIVA, D. R. Flutuação populacional de moscas-das-frutas (Diptera: Tephritidae) associadas a variedades de manga no município de José de Freitas-Piauí. Revista Brasileira de Fruticultura, v.30, n.1, p.112117, 2008.

GARCIA, F. R. M.; CAMPOS, J. V.; CORSEUIL, E. Flutuação populacional de Anastrepha fraterculus (Wiedemann, 1830) (Diptera, Tephritidae) na Região Oeste de Santa Catarina, Brasil. Revista Brasileira de Entomologia, v.47, n.3, p.415-420, 2003.

MARTINS, D. S. Manejo integrado de moscas-das-frutas. In: ZAMBOLIM, L. (Org.) Manejo integrado de fruteiras tropicais: doenças e pragas. Viçosa: UFV, 2002. p. 615-647.

MONTES, S. M. N. M.; RAGA, A.; BOLIANI, A. C.; DOS SANTOS, P. C. Dinâmica populacional e incidência de moscas-das-frutas e parasitóides em cultivares de pessegueiros (Prunus persica L. Batsch) no município de Presidente Prudente - SP. Revista Brasileira de Fruticultura, v.33, n.2, p.402-411, 2011.

MOURA, A. P.; MOURA, D. C. M. Espécies de moscas-das-frutas (Diptera: Tephritidae) associadas à cultura da goiabeira (Psidium guajava Linnaeus) em Fortaleza, Ceará. Arquivos do Instituto Biológico, v.73, n.1, p.65-71, 2006.

NASCIMENTO, A. S.; CARVALHO, R. S. Manejo Integrado de Moscas-das-frutas. In: MALAVASI, A.; ZUCCHI, R. A. (Org.) Moscas-das-frutas de importância econômica no Brasil: conhecimento básico e aplicado. Ribeirão Preto: Holos, 2000. p. 169173.

PARAJUlEE, M. N.; SLOSSER, J. E. Potential of yellow sticky traps for lady beetle survey in cotton. Journal of Economic Entomology, v.96, n.1, p.239-245, 2003.

PAZINI, W. C.; GALLI, J. C. Redução de aplicações de inseticidas através da adoção de táticas de manejo integrado do Triozoida limbata (Enderlein, 1918) (Hemiptera: Triozidae) em goiabeira. Revista Brasileira de Fruticultura, v.33, n.1, p.66-72, 2011.

RAGA, A.; DE SOUZA FILHO, M. F.; PRESTES, D. A. O.; DE AZEVEDO FILHO, J. A.; SATO, M. E. Susceptibility of guava genotypes to natural infestation by Anastrepha spp. (Diptera: Tephritidae) in the municipality of Monte Alegre do Sul, State of São Paulo, Brazil. Neotropical Entomology, v.35, n.1, p.121-125, 2006.

RAMPAZZO, E. F. Dinâmica populacional de moscas-das-frutas do gênero Anastrepha (Wiedmann) (Diptera: Tephritidae), seus 
parasitóides e predadores coletados em pomares de goiaba (Psidium guajava L.) nos municípios de Jaboticabal e Monte Alto - SP. 1994. 133 f. Dissertação (Mestrado em Entomologia) - Faculdade de Ciências Agrárias e Veterinárias, Universidade Estadual Paulista, Jaboticabal, 1994.

RONCHI-TELES, B.; SILVA, N. M. Flutuação populacional de Anastrepha Schiner (Diptera: Tephritidae) na região de Manaus, AM. Neotropical Entomology, v.34, n.5, p.733-741, 2005.
SOUZA FILHO, M. F.; RAGA, A.; ZUCCHI,

R. A. Moscas-das-frutas nos estados brasileiros: São Paulo. In: MALAVASI, A.; ZUCCHI, R. A. (Org.) Moscas-das-frutas de importância econômica no Brasil: conhecimento básico e aplicado. Ribeirão Preto: Holos, 2000. p. 277-283.

SOUZA, A. J. B.; DE LIMA, M. G. A.; GUIMARÃES, J. A.; FIGUEIREDO, A. E. Q. Moscas-das-frutas (Diptera: Tephritidae) associadas às plantas hospedeiras do pomar do Campus do Pici da Universidade Federal do Ceará. Arquivos do Instituto Biológico, v.75, n.1, p.21-27, 2008. 